\title{
Prevalência do tabagismo entre escolares de Florianópolis, SC, Brasil e as contribuições da enfermagem
}

\author{
Smoking prevalence among students from Florianópolis, SC, Brazil and nursing contributions
}

Prevalencia del tabaquismo entre estudiantes de Florianópolis, SC, Brasil e las contribuciones de la enfermería

\section{Elke Annegret Krezschmar Cordeiro', Emil Kupek', Jussara Gue Martini"}

'Universidade Federal de Santa Catarina. Pós-graduação em Saúde Pública. Florianópolis, SC

"Universidade Federal de Santa Catarina. Departamento de Enfermagem. Florianópolis, SC

Submissão: 26/02/2009

Aprovação: 29/08/2010

\section{RESUMO}

Estudo de prevalência do tabagismo entre escolares do ensino fundamental e médio, utilizando dados secundários do InQuérito Escolar "Vigescola" realizado pelo Centro de Pesquisas em Oncologia de Santa Catarina. Sendo algumas variáveis: idade, iniciação ao cigarro, nível de conhecimento sobre o tema, dentre outras. Verificou-se que 41,6\% dos escolares fumaram alguma vez na vida, sem diferença estatística entre os sexos. Entre os que já experimentaram, 38,3\% o fizeram entre 7 e 1 I anos. Quanto ao conhecimento, 92,5\% dos entrevistados consideram o cigarro prejudicial à saúde. A análise da prevalência proporcionou uma caracterização dos fatores associados, subsidiando uma prevenção mais efetiva da enfermagem e sua inserção nas escolas.

Descritores: Tabagismo; Prevalência; Fatores de risco; Enfermagem.

\section{ABSTRACT}

Study of the prevalence of smoking among school children, using secondary data from the Survey School "Doing Research - Monitoring of Smoking in School", conducted by the Centre for Oncologic Research of Santa Catarina. Included variables such as age, the starting in smoking, level of knowledge about the subject, among others. It was found that $41.6 \%$ of students smoked sometime in life, no statistical difference between the sexes. Among those who have tried, 38.3\% did so between 7 and 11 years. The analysis provided a characterization of the prevalence of risk factors, subsidizing a more effective prevention of nursing and its integration in schools.

Key words: Smoking; Prevalence; Risk factors; Nursing.

\section{RESUMEN}

Estudio de la prevalencia del tabaquismo entre los escolares de los níveles fundamental y medio, usandose los datos del Ineuerito Escolar "Vigiescola" realizado por el Centro de Investigaciones en Oncología de Santa Catarina. Algunas variables fueron la edad, iniciación al tabaquismo, nível de conocimiento sobre el tema, entre otras. Se verificó que 41,6\% de los estudiantes fumaran alguna vez en la vida, sin diferencia estadísdica entre los sexos. Entre los que han experimentado, el 38,3\% lo hicieron entre los 7 y los 11 años. Cuanto a los conocimientos, $92,5 \%$ de los encuestados creen Que el cigarrillo es perjudicial para la salud. El análisis de la prevalencia proporciono una caracterización de los factores associados, subsidiando una prevención mas efectiva de la enfermería y su integración en las escuelas.

Descriptores: Tabaquismo; Prevalencia; Factores de riesgo; Enfermería. 


\section{INTRODUÇÃO}

Dados mais recentes de mortalidade revelam que a tendência do número de mortes pelo uso frequente do cigarro, nos últimos anos, é crescente. Segundo estimativas do Ministério da Saúde, o tabagismo foi a principal causa de câncer de pulmão, com um risco atribuível a 90,0\% em nosso país ${ }^{(1)}$. Atualmente, cerca de um terço da população adulta fuma. No mesmo ano, foram estimados 15.165 e 6.920 casos novos, entre homens e mulheres, respectivamente ${ }^{(2)}$.

O Ministério da Saúde estima que no Brasil, para o ano de 2008, sendo semelhante a projeção para 2009 o número de casos novos de câncer de pulmão seja de 17.810 em homens e de 9.460 em mulheres, sendo a segunda causa de morte por localização primária entre homens e a terceira causa para mulheres, nas regiões sul, sudeste e centro oeste ${ }^{(3)}$. Fato importante a ser considerado com relação ao tabagismo é Que este é um fator de risco modificável e Que a grande maioria dos fumantes inicia o hábito de fumar na adolescência. Com base nisso, a prevenção de tabagismo em crianças e adolescentes torna-se a principal linha de atuação na prevenção de câncer na idade adulta. Historicamente a saúde do escolar vem recebendo atenção nos últimos anos ${ }^{(4)}$. No contexto da enfermagem sua inserção nas escolas ainda é muito recente, sendo anteriormente realizada pelas visitadoras sanitárias ${ }^{(4)}$. A partir da década de setenta a enfermagem atua com mais frequência nas escolas brasileiras iniciando em São Paulo sua trajetória.

Em 5 de dezembro de 2007 através do Decreto presidencial de $n^{\circ} 6.286$ foi instituído no âmbito dos Ministérios da Educação e da Saúde o "Programa Saúde na Escola" (PSE) com a intenção de contribuir para a formação integral dos escolares da rede pública de educação básica, sendo Que consta o seu artigo $4^{\circ}$, Que estas ações em devem ser desenvolvidas utilizando-se os princípios e diretrizes do SUS, atenção, promoção, prevenção e assistência, podendo compreender as seguintes ações, entre outras ${ }^{(5)}$.

Com a ênfase voltada para a Atenção Básica através da atuação nas escolas, a enfermagem pode abrir seu espaço e intensificar sua atuação. Estudos de prevalência do tabagismo realizados no Brasil e encontrados na literatura ${ }^{(6-7)}$ são de grande valia e necessários entre escolares, pois tem como objetivo conhecer a prevalência e detectar a percepção destes em relação ao hábito de fumar, no sentido de obter subsídios para uma prevenção mais efetiva do câncer. A adolescência é colocada como uma fase de experimentações, incluindo nesta iniciação o cigarro, mas Que pode ser deixado de lado se houver uma conscientização dos riscos através da mídia, das escolas, por parte das instituições governamentais de saúde. Através de avaliações periódicas para detecção precoce de doenças ditas da infância e adolescência, no âmbito escolar, o enfermeiro pode atuar como agente promotor de hábitos de vida saudáveis.

Alguns estudos realizados sobre adolescentes em Santa Catarina mostram Que o cigarro está presente na vida do escolar ${ }^{(8-9)}$. Entretanto, pouco se conhece sobre a prevalência do tabagismo no estado de Santa Catarina, principalmente na capital Florianópolis, dificultando a elaboração de estratégias de prevenção, além de poucos estudos mostrarem a inserção do enfermeiro nas escolas.

O presente artigo foi elaborado utilizando como referencia um estudo realizado em 2003 com base nos dados secundários, não publicados, a partir do Inquérito de base escolar "Fazendo Pesquisa - Vigilância de Tabagismo em Escolares (VIGESCOLA)", sendo a coleta de dados realizada em novembro e dezembro de $2002 \mathrm{em}$ Florianópolis.

A relevância deste estudo está em mostrar que dados de prevalência mostram a evidência de modos de comportamento de uma sociedade e Quanto pode ser atuado no Que se refere a prevenção de fatores de risco. Além de Florianópolis participaram do Ineuérito outras 12 capitais brasileiras no mesmo ano. $\mathrm{O}$ Instituto Nacional do Câncer (INCA), o Centro de Pesquisas Oncológicas de Santa Catarina (CEPON) e a Secretaria de Estado da Saúde de Santa Catarina (SES-SC) foram instituições envolvidas no estudo.

O objetido do estudo foi apresentar a relação entre tabagismo, sexo, idade, conhecimento e informações obtidas sobre o tema, entre estudantes do ensino fundamental e médio da cidade de Florianópolis, SC com base em registros prévios do VIGIESCOLA.

\section{MÉTODO}

Tratou-se de um estudo retrospectivo cuja população foram os registros do VIGIESCOLA, os Quais foram obtidos junto a escolares de $7^{\mathrm{a}}$ e $8^{\mathrm{a}}$ séries do Ensino Fundamental e da $\mathrm{I}^{\mathrm{a}}$ série do Ensino Médio, de escolas públicas e privadas no município de Florianópolis, Santa Catarina, no ano de 2002. Foram selecionados aleatoriamente 24 escolas com um total de 2163 alunos matriculados. A amostra aleatória das escolas e das classes dentro das escolas selecionadas foi de 288 escolares, correspondendo a 14,0\% dos alunos matriculados. Destes, foram utilizados para a amostra dados de 281 Questionários. Neste estudo descritivo de prevalência, utilizouse como variável dependente o tabagismo e como principais variáveis independentes: gênero, idade e série escolar, iniciação ao cigarro, presença de pais fumantes, forma de aQuisição dos cigarros, nível de conhecimento Que os escolares tinham sobre os malefícios do cigarro. A definição de fumante foi adotada segundo critério utilizado pelo INCA, ou seja, escolares Que fumaram cigarros em um ou mais dias nos últimos trinta dias Que antecederam a pesQuisa do VIGESCOLA.

$\mathrm{O}$ instrumento utilizado pelo inquérito de base escolar do VIGESCOLA foi um Questionário padronizado, anônimo, autoaplicado nas classes sorteadas, somente aos escolares Que apresentam o Termo de Autorização Livre e Esclarecido de Pais (ou de Guardião Legal), assinado pelo responsável. O Questionário constava de setenta perguntas fechadas. Após o preenchimento dos gabaritos, os mesmos foram recolhidos e encaminhados para o CEPON. Os dados referentes aos gabaritos utilizados neste estudo especificamente foram encaminhados para um banco de dados elaborado através do software EpiData 2.1. A análise estatística do estudo foi realizada através do programa Epi-Info 6.04d, utilizando percentuais, o teste de Qui-Quadrado de Pearson e risco relativo (com intervalo de confiança de 95\%) para significância estatística das associações entre o tabagismo e seus fatores associados.

\section{RESULTADOS E DISCUSSÃO}

Neste trabalho, apresentamos a relação entre tabagismo e gênero, idade, conhecimento Que os escolares têm sobre os 
Tabela 1. Distribuição de escolares Que já experimentaram fumar alguma vez na vida por gênero.

\begin{tabular}{|c|c|c|c|c|c|c|c|}
\hline \multirow{2}{*}{ Categoria de experimentação } & \multicolumn{2}{|c|}{ Masculino } & \multicolumn{2}{|c|}{ Feminino } & \multicolumn{2}{|c|}{ Total } & \multirow{2}{*}{$p$ valor } \\
\hline & $\mathrm{n}$ & $\%$ & $n$ & $\%$ & $\mathrm{n}$ & $\%$ & \\
\hline Experimentaram & 58 & 41,7 & 59 & 41,5 & 117 & 41,6 & \\
\hline Não experimentaram & 81 & 58,3 & 83 & 58,5 & 164 & 58,4 & 0,927 \\
\hline Total & 139 & 100,0 & 142 & 100,0 & 281 & 100,0 & \\
\hline
\end{tabular}

Tabela 2. Distribuição de escolares Que experimentaram cigarros por gênero e idade.

\begin{tabular}{lccccccc}
\hline \multirow{2}{*}{ Experimentaram fumar } & \multicolumn{2}{c}{ Masculino } & \multicolumn{2}{c}{ Feminino } & \multicolumn{2}{c}{ Total } & \multirow{2}{*}{$\boldsymbol{P}$ valor } \\
\cline { 2 - 7 } & $\mathbf{n}$ & $\mathbf{\%}$ & $\mathbf{n}$ & $\mathbf{\%}$ & $\mathbf{n}$ & $\mathbf{\%}$ & \\
Até 13 anos & 44 & 80,0 & 28 & 49,1 & 72 & 64,3 & \\
De 14 a 15 anos & 7 & 12,7 & 28 & 49,1 & 35 & 31,3 & $<0,001$ \\
De 16 anos ou mais & 4 & 7,3 & 1 & 1,8 & 5 & 4,5 & \\
\hline Total & 53 & 100,0 & 57 & 100,0 & 112 & 100,0 & \\
\hline
\end{tabular}

Tabela 3. Distribuição de escolares por gênero de acordo com o relacionamento e comportamento do grupo de amigos e o hábito de fumar.

\begin{tabular}{lccc}
\hline Opiniões dos escolares & Masculino (\%) & Feminino (\%) & RR (IC 95\%) \\
\hline Aceitam cigarros oferecidos por um amigo & 17,2 & 12,6 & $1,19(0,89--1,59)$ \\
Acreditam Que não há diferença entre fumantes e não & 67,7 & 76,5 & $0,81(0,63--1,03)$ \\
fumantes terem mais ou menos amigos & 39,5 & 54,9 & $0,73(0,57--0,93)$ \\
Acreditam Que fumar faz perder peso & 61,8 & 71,1 & $0,82(0,64--1,03)$ \\
Escolares Que tem colegas fumantes & 20,0 & 27,0 & $0,81(0,43-1,51)$ \\
Escolares fumantes Que receberam conselhos de & 47,4 & 64,7 & $0,71(0,56-0,89)$ \\
colegas para parar de fumar & 74,1 & 72,3 & $1,05(0,80-1,37)$ \\
Acham Que meninos Que fumam são menos atraentes & & & \\
Acham Que meninas Que fumam são menos atraentes &
\end{tabular}

Tabela 4. Distribuição dos escolares por gênero, de acordo com o conhecimento Que tem sobre os malefícios do consumo de cigarros.

\begin{tabular}{|c|c|c|c|c|c|}
\hline Conhecimentos dos escolares & Grau das respostas & Masculino & Feminino & $\begin{array}{c}\text { Qui- } \\
\text { Quadrado }\end{array}$ & $p$ valor \\
\hline \multirow{5}{*}{$\begin{array}{l}\text { Acham que o cigarro é prejudicial à } \\
\text { saúde }\end{array}$} & Com certeza não & 2,9 & 0,7 & \multirow{5}{*}{3,18} & \multirow{5}{*}{0,364} \\
\hline & Provavelmente não & 1,4 & 0,7 & & \\
\hline & Provavelmente sim & 5,8 & 3,5 & & \\
\hline & Com certeza sim & 89,9 & 95,1 & & \\
\hline & Com certeza não & 63,3 & 58,5 & & \\
\hline \multirow{3}{*}{$\begin{array}{l}\text { Você acha difícil largar o cigarro } \\
\text { depois de começar a fumar? }\end{array}$} & Provavelmente não & 28,8 & 32,4 & \multirow{3}{*}{6,33} & \multirow{3}{*}{0,096} \\
\hline & Provavelmente sim & 5,0 & 9,2 & & \\
\hline & Com certeza sim & 2,9 & 0,0 & & \\
\hline \multirow{3}{*}{$\begin{array}{l}\text { Percebem a nicotina como uma } \\
\text { droga Que causa dependência }\end{array}$} & Sim & 93,5 & 98,6 & \multirow{3}{*}{5,17} & \multirow{3}{*}{0,075} \\
\hline & Não & 5,0 & 0,7 & & \\
\hline & Ignorado & 1,4 & 0,7 & & \\
\hline \multirow{3}{*}{$\begin{array}{l}\text { Acreditam Que mulheres grávidas } \\
\text { possam fumar sem prejudicar o feto }\end{array}$} & Sim & 12,9 & 9,2 & \multirow{3}{*}{2,92} & \multirow{3}{*}{0,232} \\
\hline & Não & 87,1 & 89,4 & & \\
\hline & Ignorado & 0,0 & 1,4 & & \\
\hline \multirow{3}{*}{$\begin{array}{l}\text { Quem fuma mais cigarros tem maior } \\
\text { chance de contrair câncer }\end{array}$} & Sim & 55,2 & 57,7 & \multirow{3}{*}{1,88} & \multirow{3}{*}{0,390} \\
\hline & Não & 47,5 & 41,5 & & \\
\hline & Ignorado & 0,0 & 0,7 & & \\
\hline \multirow{2}{*}{$\begin{array}{l}\text { Tem noção Que a fumaça ambiental } \\
\text { causa várias doenças inclusive a do } \\
\text { coração }\end{array}$} & Sim & 899 & 923 & \multirow[b]{2}{*}{0,47} & \multirow[b]{2}{*}{0,494} \\
\hline & Não & 10,1 & $\begin{array}{l}32,3 \\
7,7\end{array}$ & & \\
\hline \multirow{4}{*}{$\begin{array}{l}\text { Acham prejudicial ser fumante } \\
\text { passivo }\end{array}$} & Com certeza não & 6,5 & 2,1 & \multirow{4}{*}{7,04} & \multirow{4}{*}{0,133} \\
\hline & Provavelmente não & 0,7 & 2,1 & & \\
\hline & Provavelmente sim & 15,8 & 10,6 & & \\
\hline & Com certeza sim & 77,0 & 84,5 & & \\
\hline
\end{tabular}


malefícios do consumo de cigarros, se receberam ou não conselhos para deixar de fumar, assim como as opiniões dos escolares sobre atrativos de fumar. Faremos uma reflexão acerca das contribuições da enfermagem, o espaço, a oportunidade Que o enfermeiro, enQuanto estudante, profissional e professor de Enfermagem tem para intervir nas ações de enfermagem na área do câncer, como agente de prevenção no âmbito escolar.

A prevalência da experimentação do cigarro encontrada foi de 41,6\% e praticamente idêntica Quanto ao gênero (Tabela 1).

Quase dois terços dos escolares experimentou fumar até os 13 anos de idade, mas as meninas iniciaram a experimentação mais tarde, sendo essa diferença estatisticamente significativa.

Entre os escolares fumantes 53,6\% comprou seus cigarros. Entre os Que tentaram comprar $87 \%$ comprou cigarros sem impedimento, mesmo sendo a venda de cigarros proibida, para menores de 18 anos no Brasil(10). De acordo com o Estatuto da Criança e do Adolescente é proibido vender produtos cujos componentes causem dependência física ou psíeuica, incluindo cigarros e bebida alcoólica para menores de idade ${ }^{(1)}$. Outra prática ilegal é o recebimento de cigarros fornecidos gratuitamente por representantes da indústria de tabaco, relatada por 5,7\% dos escolares entrevistados.

Após verificar Que a associação entre experimentação de fumar e o hábito de fumar dos pais foi estatisticamente significativa, calculou-se o risco relativo de várias categorias de exposição usando como referência a porcentagem de fumantes entre os escolares Que responderam Que seus pais não fumam $(57 / 117=32,76 \%)$. Quando pai e mãe fumam, o risco de filhos também ter experimentado o cigarro é o dobro Que Quando nenhum dos pais fuma, com intervalo de confiança (IC) de $95 \%$ de 1,40 a 2,84 . O risco é um pouco menor Quando somente um dos pais fuma: caso seja a mãe, o risco aumenta 1,76 (IC de 95\% entre 1.19 e 2.61) vezes; caso seja o pai, o risco aumenta 1.41 (IC de 95\% entre 0.98 e 2,02) vezes.

Vale a pena ressaltar Que $92,5 \%$ dos escolares sabem Que o cigarro é prejudicial para a saúde $60,9 \%$ acham difícil largar o cigarro depois de começar a fumar e $96,1 \%$ percebe a nicotina como uma droga Que causa dependência, 91 , 1\% sabe Que a fumaça do cigarro no ambiente pode causar várias doenças e $94,0 \%$ sabe Que ser fumante passivo é prejudicial para a saúde. De uma forma geral, os escolares se mostraram conscientes dos malefícios do cigarro, não havendo diferenças estatisticamente significativas com relação ao gênero.

Entretanto cabe um alerta, pois de acordo com a pesquisa da metade dos escolares fumantes não recebeu conselhos para abandonar o hábito. Entre os Que receberam esse conselho, destaca se a influencia de seus pares. Somente $7,7 \%$ dos fumantes do sexo masculino gostariam parar de fumar, comparado com $72.7 \%$ do sexo feminino, sendo essa diferença estatisticamente significativa ( $p=0.00$ I no teste de Qui-Quadrado).

Entre as opiniões pesquisadas, observou-se a diferença estatisticamente significativa nos dois itens: os meninos são menos propensos a acreditar Que fumar emagrece e Que meninos Que fumam são menos atraentes (Tabela 4). Também é interessante observar que a chance de os meninos acharem meninos fumantes menos atraentes é $40 \%$ (intervalo de confiança de $95 \%$ entre $24 \%$ e 53\%) menor que no caso das meninas fumantes.

$\mathrm{Na}$ Tabela 4 Quando foram associadas as variáveis sobre o conhecimento Que o escolar tem sobre os malefícios do cigarro, verificou-se Que: $92,5 \%(n=260)$ acham Que o cigarro é prejudicial para a saúde; $60,9 \%(n=171)$ acham difícil largar o cigarro depois de começar a fumar e $96,1 \%(n=270)$ percebem a nicotina como uma droga Que causa dependência.

Para o início da experimentação do cigarro entre a préadolescência e adolescência, ou seja, entre 11 e 15 anos, a prevalência encontrada em Florianópolis vem de encontro com as Que foram encontradas em estudos de outras regiões do país e do mundo ${ }^{(12,7)}$. Em Santiago, no Chile, a experimentação com fumo de cigarro ocorre tipicamente na faixa etária entre 13 e 15 anos, não havendo diferença estatística entre os gêneros ${ }^{(13)}$. A prevalência do uso do cigarro alguma vez na vida em escolares de Florianópolis foi de 41,3\%. Dados semelhantes foram evidenciados numa escola pública de Florianópolis em $1997^{(9)}$. A prevalência encontrada em Blumenau em 2002 foi semelhante ${ }^{(8)}$. A prevalência de Florianópolis também pode ser comparada a algumas capitais do país Que no ano de 2002 realizaram a mesma pesquisa do VIGESCOLA ${ }^{(6)}$. Dentre as cidades com percentuais semelhantes, podemos citar São Luis, João Pessoa, Boa Vista e Natal.

A prevalência do tabagismo em Florianópolis evidenciou uma prevalência de 12,9\% (Tabela 3), semelhante a outros estudos nacionais e internacionais. Um estudo feito em Ribeirão Preto (SP) encontrou uma prevalência de adolescentes fumantes um pouco acima do encontrado em Florianópolis ${ }^{(7)}$. Um outro estudo sobre tabagismo em escolares realizado por Ivanovic na cidade de Santiago do Chile, encontrou uma prevalência semelhante ${ }^{(13)}$.

Quanto a iniciação ao cigarro, chama atenção Que $80 \%$ dos rapazes e Quase a metade das meninas escolares de Florianópolis já experimentaram fumar até os 13 anos. O mesmo resultado foi encontrado nas outras capitais brasileiras, e deve servir para uma alerta: as mulheres estão começando a fumar mais cedo. Também vale a pena destacar Que, em Florianópolis, um terço dos Que experimentaram fumar alguma vez na vida tornou-se fumante regular. Neste sentido, a escola e as equipes de Saúde, incluindo-se e Enfermagem nas escolas através do Programa Saúde na Escola tem um papel de destaque na prevenção de tabagismo entre os adolescentes.

Quanto aos pais fumantes, o percentual ainda é bem significativo, verificando-se a associação da experimentação e o habito de fumar dos pais. Cabe um alerta, pois a criança e o adolescente tornam-se fumantes passivos, além de verem no exemplo dos pais um comportamento prejudicial à saúde. Os resultados da VIGESCOLA nas outras capitais brasileiras confirmaram esse achado de Florianópolis.

Quanto ao conhecimento Que os escolares de Florianópolis tem sobre os malefícios do cigarro, uma resposta semelhante foi encontrada na cidade de Amparo, $\mathbf{S P}^{(14)}$. A maioria dos escolares acredita Que o cigarro faz mal para a saúde, entretanto a prevalência de experimentação e de fumantes regulares é elevada. Outro ponto importante a ser considerado nas ações de prevenção, é fundamental para Que o adolescente possa entender melhor a razão exata de por Que não fumar. Os significados, as sensações e o imaginário social deste escolar podem contribuir para as ações de prevenção.

A análise dos dados referentes à obtenção dos cigarros mostrou Que entre os escolares que fumam, boa parte compra seus cigarros. 
Mais preocupante ainda, entre aQueles Que compram seus próprios cigarros, a maioria não encontrou dificuldades em adQuiri-los, apesar de serem menores de idade. Dados semelhantes foram encontrados no estudo do VIGESCOLA realizado em outras doze capitais brasileiras $^{(7)}$.

As meninas mais acreditam Que fumar faz perder peso. A ênfase na magreza Que muitos modelos femininos destacam pode ter contribuído para tal fato. Também é interessante observar Que $47,4 \%$ dos meninos acham meninos fumantes menos atraentes, comparado com $74,1 \%$ das meninas fumantes, ou seja, a rejeição é significativamente maior no último caso. As meninas têm a mesma direção de viés de julgamento, mas com uma magnitude bem menor.

Embora o estudo realizado em Florianópolis tenha seguido uma metodologia já testada e aprovada, com instrumentos padronizados e amostragem aleatória dos escolares, é bom considerar algumas limitações do estudo. O número de Questionários respondidos foi inferior ao esperado, comparado ao número de escolares matriculados na rede de ensino de Florianópolis, sendo o fator que mais contribuiu para isso Que a maioria dos escolares não trouxe o termo de consentimento assinado pelos pais ou responsáveis Quando da aplicação do Questionário. A omissão do consentimento pode indicar um fator de confusão, caso tivesse relacionado com hábito de fumar dos escolares. É concebível que os alunos Que trouxeram o consentimento têm melhores relações com os pais, o que é reconhecido enquanto fator de prevenção. Estudos deste tipo podem contribuir para responder as Questões com relação a essas limitações, principalmente se forem realizados de um modo regular, permitindo monitorar as tendências do hábito de fumar e seus fatores de risco. Torna-se fundamental a atuação da equipe de saúde na prevenção do tabagismo entre escolares, ampliando-se a atenção para professores e pais no sentido de mostrar as evidências estatís- ticas e baseado nestes dados propiciar meios Que possam desenvolver estratégias para a diminuição desta prevalência ${ }^{(15-16)}$.

\section{CONCLUSÕES}

A prevalência da experimentação de cigarro de 41,3\% entre escolares em Florianópolis é alta, apesar de semelhante a outras capitais no nosso país. A prevalência de fumantes de 12,8\% ficou pouco abaixo da média nacional.

Os meninos iniciam o hábito de fumar mais cedo, entretanto as meninas estão fumando mais na faixa etária de 14-15 anos, com um percentual de 49,1\%. A maioria dos escolares tem noção dos malefícios do cigarro, apesar do número de fumantes ser elevado. A educação para a saúde e Qualidade de vida dever ser colocada em pauta nos setores educacionais.

Dentro do Programa Saúde na Escola, o enfermeiro tem papel fundamental como integrante da equipe de saúde no sentido de fornecer possibilidades de compreensão entre os escolares dos prejuízos Que o cigarro pode trazer para sua saúde, entretanto, os estudos de prevalência mostram Que ainda há muito a ser feito, visto Que a influência dos colegas é evidente no estímulo para ser um provável fumante já no período escolar inicial.

Cabe como sugestão um recurso importante Que é a atuação dos acadêmicos de enfermagem, Que utilizam a Atenção Básica enQuanto espaço para campo de estágio. Os enfermeiros enQuanto educadores podem tornar possível sua inserção no ambiente escolar estimulando intervenções, utilizando estratégias criativas Que possam ser aplicadas na vida diária, e incorporadas no cotidiano destes escolares além de abrir um campo maior de interesse profissional futuro pela vida do escolar por parte dos acadêmicos de enfermagem.

\section{REFERÊNCIAS}

I. Ministério da Saúde (BR). Instituto Nacional do Câncer. Estimativas da incidência e mortalidade por câncer no Brasil. Rio de Janeiro: Instituto Nacional do Câncer; 2002.

2. Ministério da Saúde (BR). Instituto Nacional do Câncer. Falando de tabagismo. Rio de Janeiro: Instituto Nacional do Câncer; 2002.

3. Ministério da Saúde (BR). Instituto Nacional do Câncer. Estimativas da incidência e mortalidade por câncer no Brasil. Rio de Janeiro: Instituto Nacional do Câncer; 2008.

4. Ferriani MGC. A inserção do enfermeiro na saúde escolar. Ribeirão Preto: Editora da Universidade de São Paulo; 1991.

5. Ministério da Saúde (BR). Programa Saúde na Escola-PSE. Decreto $n^{\circ} 6.286$ de 5 de dezembro de 2007. Brasília: Ministério da Saúde; 2007.

6. Ministério da Saúde (BR). Instituto Nacional do Câncer. Vigescola: Vigilância de tabagismo em escolares. Dados e fatos: 12 doze capitais brasileiras: Rio de Janeiro: Instituto Nacional do Câncer; 2004.

7. Muza GM. Consumo de substâncias psicoativas por adolescentes escolares de Ribeirão Preto, SP (Brasil). II Distribuição do consumo por classes sociais. Reva Saúde Pública 1997; 31(2): 163-70.
8. Novaes AJ. Estilo de vida relacionado à saúde e hábitos comportamentais em escolares do ensino médio do município de Blumenau, SC: a influência da escola [tese]. Florianópolis: Universidade Federal de Santa Catarina; 2002.

9. Baus I, Kupek E, Pires M. Prevalência e fatores de risco relacionados ao uso de drogas entre escolares. Rev Saúde Pública 2002; 36(1): 40-6.

10. Ministério da Saúde (BR). Legislação Federal de Controle do Tabagismo. Rio de Janeiro: Instituto Nacional do Câncer; 2004.

11. Ministério da Justiça (BR). Estatuto da Criança e do Adolescente.Lei no. 8069 de 13 de julho de 1990 . Brasília: Ministério da Justiça; 1990.

12. Laniado RL, Moreno C, Woodruff SI, Vargas R, Sallis JF, Elder IP. TabaQuismo en ninõs y adolescentes de la ciudad de Tijuana, Mexico. Bol Med Hospital Infantil Mexico 1994; 5 I (8): 52833.

12. Ivanovic DM. Factores Que incidem en el habito de fumar de escolares de educacion basica y media del Chile. Rev Saúde Pública 1997; 3 I (1): 30-43.

13. Moraes MA, Rodrigues SSF, Barbosa MAB, Cavalcante ZO. Estudo da prevalência do tabagismo em escolares do $1^{\circ}$ e $2^{\circ}$ Graus. A criança e o adolescente como agente de mudança 
desse hábito, no município de Amparo-SP. Rev Bras Enferm 1991; 44(1): 60-9.

14. Freitas LV, Freitas GL, Lopes EM, Aguiar IR, Falcão Júnior SP, Rabelo STO, et al. Prevenção de tabagismo em escolares: um relato de experiência. In: Anais da $57^{a}$ Reunião Anual da SBPC,
2005 jul; Fortaleza (CE), Brasil. [citado em 2008 Abril 4]. Disponível em: http://www.sbpcnet.org.br/livro/57ra/programas/ senior/RESUMOS/resumo_1165.html.

15. Associação Brasileira de Enfermagem. Adolescer: compreender, atuar, acolher. Projeto Acolher. Brasília: ABEn; 2001 . 